\title{
Management of acetabulum fracture with contralateral neck of femur in an obese patient: a rare case report
}

\author{
Sidharth D.*, Thyagarajan U., Raghavendran B., Raghul Siddharth
}

\begin{abstract}
Department of Orthopedics, Sri Ramachandran Institute of Higher Education and Research, Chennai, Tamil Nadu, India
\end{abstract}

Received: 26 November 2021

Accepted: 03 February 2022

*Correspondence:

Dr. Sidharth D,

E-mail: sidharth.dileep3@gmail.com

Copyright: (C) the author(s), publisher and licensee Medip Academy. This is an open-access article distributed under the terms of the Creative Commons Attribution Non-Commercial License, which permits unrestricted non-commercial use, distribution, and reproduction in any medium, provided the original work is properly cited.

\begin{abstract}
Acetabular fractures are complex fractures that pose a challenge for orthopaedic trauma surgeons due to their extension into the hip joint. The simultaneous occurrence of contralateral neck of femur fracture in an obese patient makes postoperative wound healing and rehabilitation even challenging. Here, we report a case of management of acetabulum fracture with contralateral neck of femur fracture in an obese patient. We are reporting a case of a 50-year-old obese female from Chennai who presented to the Department of Orthopaedics, SRIHER, Chennai, with complaints of bilateral hip pain and inability to weight bear on bilateral lower limb, following a high-velocity road traffic accident. She was diagnosed to have left acetabulum fracture with right hip displaced neck of femur fracture. We managed her with right hip bipolar hemiarthroplasty and left acetabulum open reduction and internal fixation. 6 month follow up showed excellent radiological and functional outcome. This case report highlights how obesity alters the surgical and medical management in obese patients with contralateral acetabulum and neck of femur fracture and the importance of a multidisciplinary approach in such polytrauma patients.
\end{abstract}

Keywords: Acetabulum, Neck of femur, Obesity, Infection

\section{INTRODUCTION}

Acetabular fractures with an incidence of approximately 3 per 1,00,000 patients per year are commonly seen in high velocity trauma. Despite the difficulty in obtaining anatomic reductions, the treatment of displaced intraarticular fractures has evolved to meet a standard of anatomic reduction with rigid internal fixation., ${ }^{1,2}$ Acetabular fractures may have catastrophic outcomes in poly trauma patients with added comorbidities like obesity. ${ }^{3}$ The concomitant occurrence of contralateral neck of femur fracture in an obese patient makes the management, post operative wound care and rehabilitation a challenging one. Herein, we describe a rare case report of management of acetabulum fracture with contralateral neck of femur fracture in an obese patient.

\section{CASE REPORT}

A 50-year-old female from central Chennai was brought to emergency with complaints of bilateral hip pain and inability to weight bear on bilateral lower limb following a high-velocity road traffic collision. There was no history of associated head injury or abdominal trauma. Patient was haemodynamically stable at initial presentation. General examination revealed the patient as obese (BMI- 32). Clinically, the right lower limb appeared to be shortened with diffuse tenderness over bilateral hips and proximal $1 / 3$ rd of right thigh and restricted movements of bilateral hips. Neurovascular status of bilateral lower limb was intact. X-ray pelvis with both hips (Figure 1) revealed fracture of anterior and posterior column, disruption of teardrop, comminuted and displaced fracture of ischial and pubic bone, pubic rami fracture, displaced fracture of 
greater trochanter, with displaced right neck of femur fracture (Garden stage III).

CT Pelvis with 3D reconstruction (Figure 2) revealed comminuted displaced fracture of left acetabulum (Judet and Letournel -Anterior column with posterior hemi transverse), comminuted displaced fracture of left ischial and pubic bone, left pubic rami, comminuted and displaced fracture of sacrum, linear mildly displaced fracture of superomedial aspect of bilateral iliac bones, comminuted displaced fracture of left greater trochanter, linear displaced fracture of right femoral neck.

She was put on bilateral lower limb foam skin traction, started on chemical DVT prophylaxis and shifted to ward. Due to recumbent position in bed with associated obesity, she developed dyspnea and desaturation.

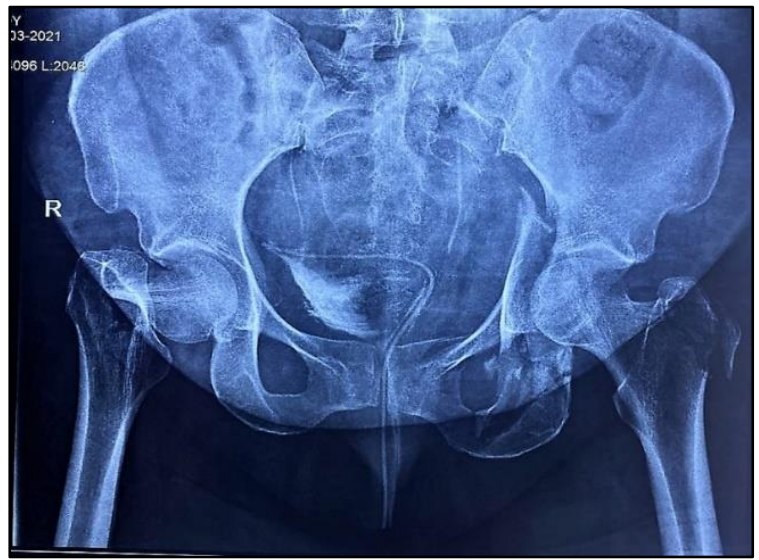

Figure 1: Xray pelvis with both hips AP viewshowing left acetabulum fracture and displaced right neck of femur fracture.

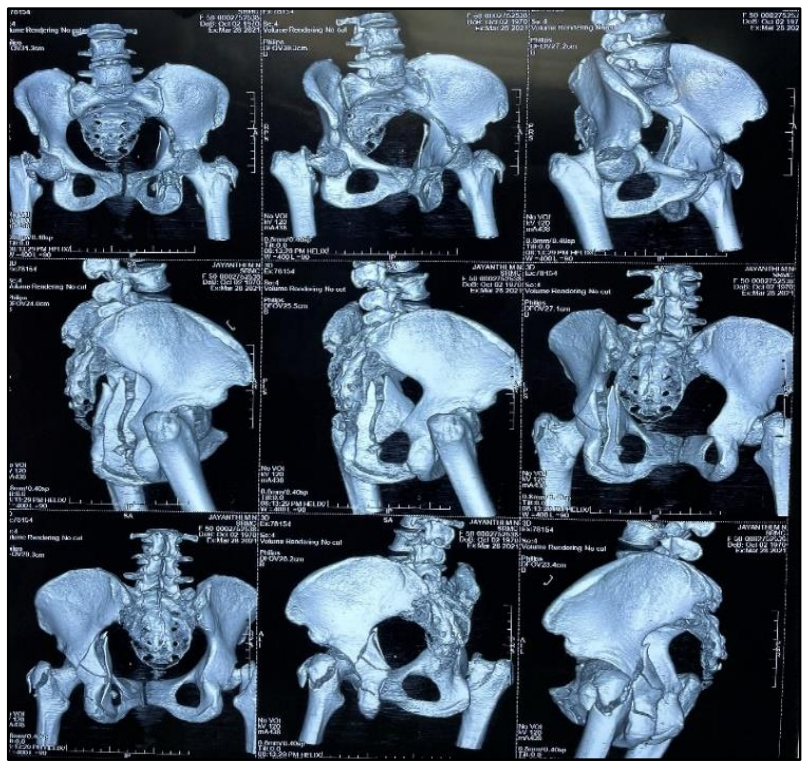

Figure 2: CT Pelvis with both hips 3D reconstruction.

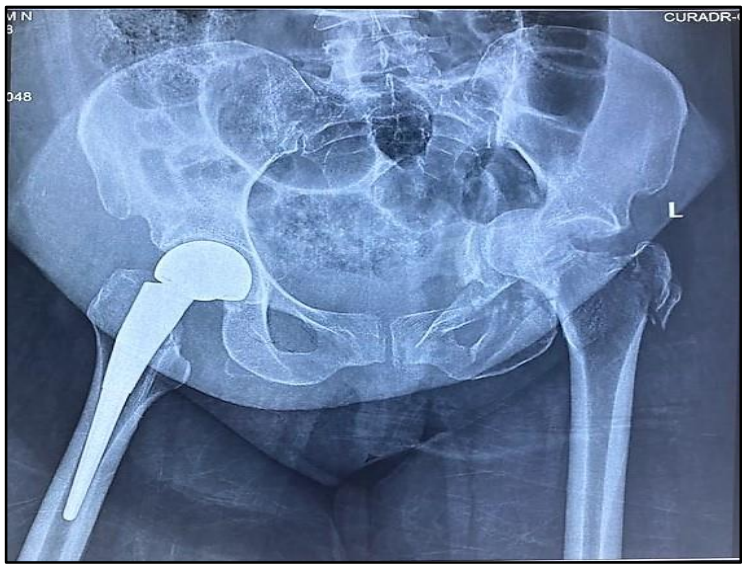

Figure 3: Postoperative right hip hemiarthroplasty $x$ ray pelvis with both hips showing bipolar prosthesis over right hip and intra pelvic migration of left femoral head with acetabulum fracture.

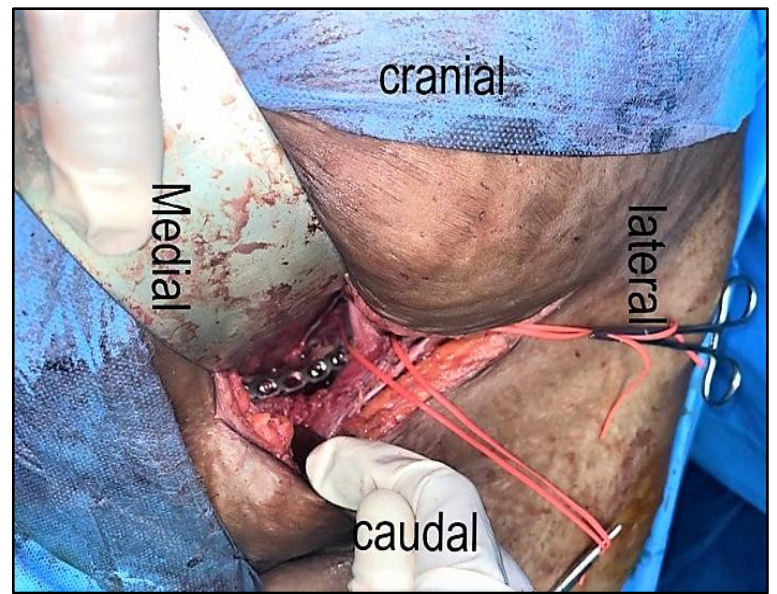

Figure 4: Modified Stoppa approach- for left acetabulum fracture showing Synthes 11hole plate inserted through the lateral window of the approach.

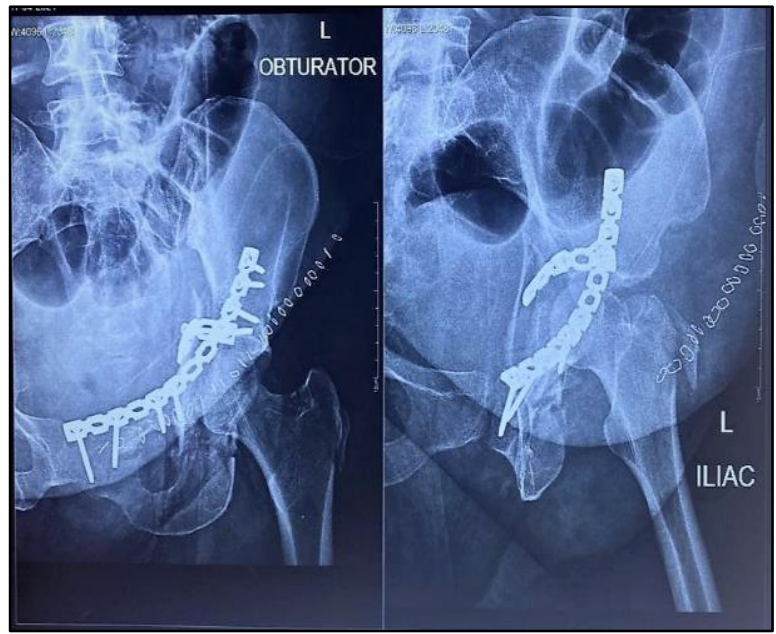

Figure 5: Post operative left Acetabulum fixation xray- Judet views. 


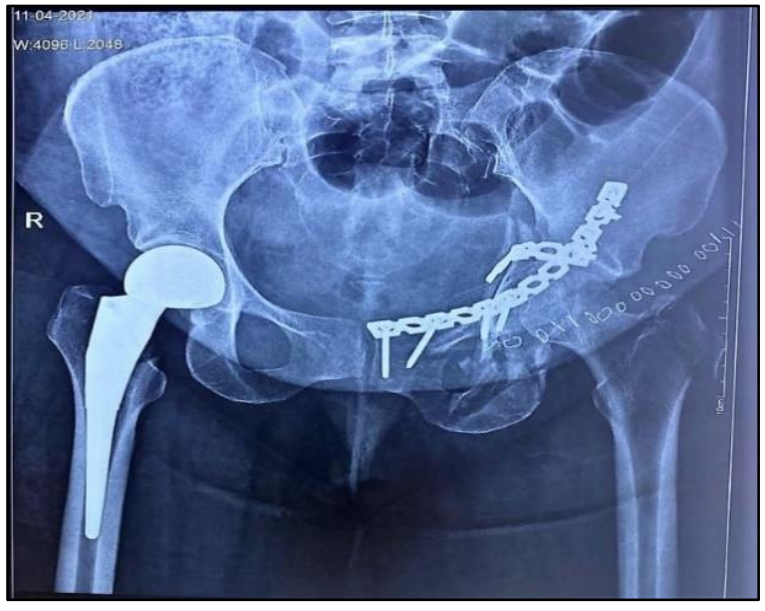

Figure 6: Immediate post operative left acetabulum fixation x-ray.

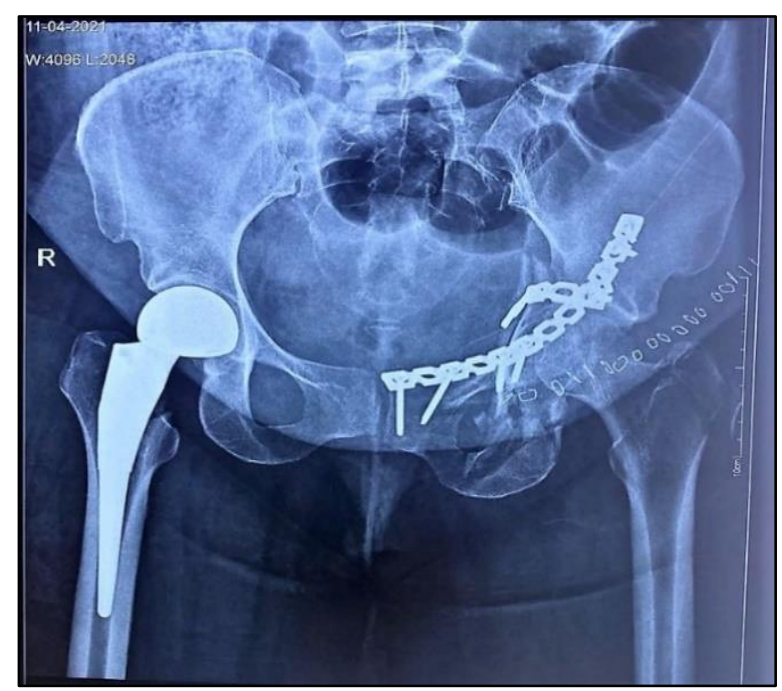

Figure 7: 6 months postoperative $x$-ray of pelvis with both hips showing good acetabular fracture union and no loosening of bipolar prothesis.

She was shifted to ICU where she put on a reclined position and started on non-invasive ventilation. After obtaining anesthetic fitness, she underwent Right hip bipolar hemiarthroplasty, under general anesthesia. She was put on left lateral position. Through Modified Hardinge approach, right hip bipolar hemiarthroplasty was done with Zimmer-Biomet modular taper lock prosthesis with femoral stem - size 4 , femoral head- $42 \mathrm{~mm}$. Postoperatively right lower limb was maintained in abduction and left leg foam skin traction was applied. She was shifted to ICU for further management and was started on IV antibiotic.

Postoperative x-ray (Figure 3) revealed proper positioning of right hip bipolar implant with good femoral stem positioning. X-ray showed intrapelvic migration of left femoral head, which arised out of intraoperative left lateral positioning, for which she was put on left leg foam skin traction according to her body weight. After 7 days of initial surgery, anesthetic fitness was obtained and she was taken up for left acetabulum fixation. Under spinal and epidural anesthesia, patient in supine position with approximately 20 degree of body roll to left side. Via modified stoppa approach (Figure 4) fracture site identified, posterior spike was fixed with $3.5 \mathrm{~mm}$ Synthes 4hole reconstruction plate and 2 appropriately sized screws. Anterior column was fixed using $3.5 \mathrm{~mm}$ Synthes 11 hole reconstruction plate and appropriately sized screws.

Postoperatively she was shifted to ICU for post-anesthetic care. She underwent serial wound inspections and dressings. Postoperative $\mathrm{X}$-ray done revealed good reduction of anterior column and the posterior spike (Figure 5, 6). She was started on right knee controlled passive flexion and extension exercises and left hip abduction and knee flexion as tolerated. She was discharged and regularly followed up for a period of 6 months.

1st and 2nd week of follow up showed wound gaping and discharge from the left ilioinguinal wound site, pus culture sensitivity was done and was started on appropriate antibiotics. Serial wound inspections showed good wound healing. She was started on guarded weight bearing on 4th week. Serial follow up x-rays revealed good fracture union and improved bilateral hip range of movements. She was started on full weight bearing mobilisation with walker support on 10th week. Postoperative follow up of 6 months revealed good fracture union (Figure 7) and excellent functional status.

\section{DISCUSSION}

Acetabulum and neck of femur fractures most frequently result from high-energy trauma. Operative treatment of acetabular fracture once decided, needs to be modified based on patient factors such as associated injuries, soft tissue condition and medical comorbidities. Obesity particularly is a comorbidity that affect nearly every organ system. Orthopaedic surgeons should be aware of the altered physiology of these patients and adjustments must be made accordingly not to compromise the haemodynamic stability. The initial assessment and evaluation of acetabular fracture in obese patients are made difficult by the excess panniculus. Standard ATLS guidelines and resuscitation protocols should be followed. Use of fluids and blood products should be done cautiously not to compromise the cardiac function.

Our case had a dense panniculus which made the examination of pelvis and lower abdomen difficult. All skin folds including the panniculus, were examined because they can hide wounds. Radiographic evaluation is important in understanding the anatomy of acetabulum and proximal femur fractures. However, radiographic evaluation in obese patient is difficult because of dense adipose tissue causing inadequate penetration of the $\mathrm{x}$ rays, resulting in poor image quality. Hence, CT scan with 
3D reconstruction helps understand fracture patterns and for preoperative planning. ${ }^{10}$ In our case, we did X-ray pelvis with both hips and $\mathrm{CT}$ pelvis with $3 \mathrm{D}$ reconstruction for better understanding of fracture pattern, which revealed comminuted displaced fracture of left acetabulum (Judet and Letournel -Anterior column with posterior hemi transverse fracture) and displaced right neck of femur fracture.

The bioavailability of antibiotics and anaesthetic medications in obese patients is affected by the increased amount of adipose tissue, simply increasing the dosing of antibiotics in turn can lead to toxicity. ${ }^{4} \mathrm{We}$ followed standard pre-procedural infection control strategies of appropriate weight based antibiotic administration 30 minutes prior to incision. Surgical fixation of right neck of femur fracture was done first with patient in left lateral position. Zimmer Biomet cementless modular bipolar taper lock system was used keeping in mind the delayed mobilisation due to the contralateral acetabular fracture and also easier conversion to total hip replacement in future. Modular Bipolar prosthesis provides improved functional outcome by better primary anchorage of the prosthesis. Cementless prosthesis has advantage of easier conversion into total hip replacement. ${ }^{9}$

In our case, left lateral positioning for hemiarthroplasty resulted in intrapelvic migration of left femoral head, for which left leg foam skin traction was applied with weights in accordance to the body weight of patient. Larger amount of weight should be applied cautiously because of risk of soft tissue and nerve injury. ${ }^{3}$ Skeletal pin traction was avoided as it is associated with greater soft tissue damage during insertion, increasing the risk of a pin tract infection, moreover the larger soft tissue envelope due to obesity makes it difficult for pin to clear the tract and thereby decreasing the working length of pin. ${ }^{4}$

Obese patients are prone to falling off during positioning, bean bags and straps should be placed to prevent this. ${ }^{3}$ Although the fracture pattern and ease of surgery decides the patient positioning, it should be kept in mind the altered physiology in obese patients and the effects of these positions as a stress riser. Lateral decubitus is best tolerated by obese patients, because the weight of panniculus is off the abdomen, allowing for greater diaphragmatic excursion. ${ }^{3}$ Due to the right hip arthroplasty, we positioned our patient in supine with 20 degree right lateral roll. To provide better access to operative field, panniculus was secured with adhesive tapes to opposite side of table. It is recommended that the patient's ventilatory status should be observed before surgery, because the extra weight of abdomen on the chest may obstruct ventilation, demanding release of the panniculus strapping. ${ }^{3}$ Keeping in mind the risk of cardiopulmonary complications, we positioned our patient in supine with 20 degree of body roll to right side and minimised the use of blankets and sheets to avoid mechanical stress on deeper tissues. We maintained adequate perfusion and oxygenation during the procedure which further helped in reducing risk of pressure sores. ${ }^{3}$

For better articular surface restoration, open reduction and internal fixation is often mandated in patients with displaced acetabular fractures. To facilitate this, the surgical approach and instrumentation should allow adequate bony visualisation. The commonly preferred approaches in obese patients include Pfannenstiel approach, ilioinguinal approach and Stoppa approach. We preferred modified stoppa approach owing to its excellent visualisation of the pelvic ring, facilitating utilisation of improved reduction and fixation and decrease the rate of complications. ${ }^{5}$ Incision length was minimal and care was taken to avoid incisions at panniculus folds, dissection through adipose layers were sharp to decreases postoperative lipolysis and postoperative wound drainage. For better handling of adipose tissue and bony visualisation, we used larger and deeper retractors. ${ }^{3}$ Soft tissue handling was done cautiously, and the surgical time was restricted to 2 hours.

The risk of infections in obese patients is multifactorial, with contributions from patient physiology, body habitus, surgical time and the trauma itself. Poor vascularity of adipose tissue results in suboptimal tissue perfusion causing compromised wound healing. ${ }^{6}$ The large surgical area of exposure in obese patients increases pressure on wound edges by the adipose tissues, further decreasing blood flow and delaying wound healing. ${ }^{7}$ In addition, intraoperative factors such as prolonged operative time, the requirement of prolonged and forceful retraction of adipose tissue results in more tissue trauma, further aggravating the chances of infection. ${ }^{8}$

Serial postoperative wound inspection was done and was found to be healthy during the period of stay in hospital. She was started on in bed mobilisation, left hip abduction and left knee flexion exercises as tolerated. Follow up wound examinations were done on an outpatient basis, revealed gaping of the acetabulum surgical wound with discharge. Culture sensitivity was obtained from wound site and was started on appropriate antibiotic. Surgical staples were removed on POD 20. She was started on guarded weight bearing on 4th week. Serial follow up Xrays revealed good fracture union and improved bilateral hip range of movements. She was started on full weight bearing mobilisation with walker support on 10th week. She was able to resume her day- day activities without any aid at the end of 12th week. Outcome assessment was done based on radiological evidence of fracture union and Harris Hip score. On the 3rd postoperative month fracture union was found to be satisfactory and had a fair Harris hip score of 77 and 76 on right and left hip respectively. 6 months after reconstruction, she did not experience any restriction in activities and was very satisfied with the outcome. Radiograph (Figure 7) demonstrated good acetabular fracture union and no loosening of prosthesis was noted. 6 months postoperatively she had an excellent Harris Hip score of 90 bilaterally. 


\section{CONCLUSION}

Management of obese polytrauma patients should be individualised. Initial management of these patients should follow standard ATLS protocols. Preoperative, intraoperative and postoperative planning plays a crucial role in management of these patients. Patient positioning, surgical approaches, soft tissue handling and instrumentations all add to the outcomes of surgery. Modular bipolar prothesis in patients with neck of femur fracture with contralateral acetabulum fracture helps decreasing surgical time and gives option of total hip replacement on later date. Postoperative physiotherapy and rehabilitation play an important role in functional outcomes in these patients.

Funding: No funding sources

Conflict of interest: None declared

Ethical approval: Not required

\section{REFERENCES}

1. Sardesai NR, Miller MA, Jauregui JJ, Griffith CK, Henn RF, Nascone JW. Operative management of acetabulum fractures in the obese patient: challenges and solutions. Orthopedic research and reviews. 2017;9:75-81.

2. Porter SE, Graves ML, Maples RA, Woodall J Jr, Wallace JG, Russell GV. Acetabular fracture reductions in the obese patient. $\mathrm{J}$ Orthop Trauma. 2011;25(6):371-7.

3. Gettys FK, Russell GV, Karunakar MA. Open treatment of pelvic and acetabular fractures. Orthop Clin North Am. 2011;42(1):69-83.

4. Lee JB, Winstead PS, Cook AM. Pharmacokinetic alterations in obesity. Orthopedics. 2006;29:984e8.

5. Cole JD, Bolhofner BR. Acetabular fracture fixation via a modified Stoppa limited intrapelvic approach. Description of operative technique and preliminary treatment results. Clin Orthop Relat Res. 1994;(305):112-23.

6. Rosell S, Belfrage E. Blood circulation in adipose tissue. Physiol Rev. 1979;59:1078e-4.

7. Armstrong D, Bortz P. An integrative review of pressure relief in surgical patients. AORN J. 2001;73:645e53.

8. Gong EM, Orvieto MA, Lyon MB. Analysis of impact of body mass index on outcomes of laparoscopic renal surgery. Urology. 2007;69:38e43.

9. Canturk Z, Canturk NZ, Cetinarslan B. Nosocomial infections and obesity in surgical patients. Obes Res. 2003;11:769e75.

10. Uppot RN. Impact of obesity on radiology. Radiol Clin North Am. 2007;45(2):231-46.

Cite this article as: Sidharth D, Thyagarajan U, Raghavendran B, Siddharth R. Management of acetabulum fracture with contralateral neck of femur in an obese patient: a rare case report. Int J Res Orthop 2022;8:278-82. 